\section{Cureus}

\title{
Anti-inflammatory and Antioxidant Effects of Sesame Oil on Atherosclerosis: A Descriptive Literature Review
}

\author{
Edmund $\mathrm{Hsu}^{1}$, Sam Parthasarathy ${ }^{2}$ \\ 1. Emergency Medicine, Mount Sinai St. Luke's - Roosevelt Hospital Center, New York, USA 2. Burnett \\ School of Biomedical Sciences, University of Central Florida College of Medicine, Orlando, USA
}

$\square$ Corresponding author: Edmund Hsu, edmund.hsu@mountsinai.org

Disclosures can be found in Additional Information at the end of the article

\section{Abstract}

Sesame oil (SO) is a supplement that has been known to have anti-inflammatory and antioxidant properties, which makes it effective for reducing atherosclerosis and the risk of cardiovascular disease. Due to the side effects of statins, the current recommended treatment for atherosclerosis and cardiovascular diseases, the idea of using dietary and nutritional supplementation has been explored. The benefits of a dietary health regime have piqued curiosity because many different cultures have reaped health benefits through the ingredients in their cooking with negligible side effects. The purpose of this literary review is to provide a broad overview of the potential benefits and risks of $\mathrm{SO}$ on the development of atherosclerosis and its direction toward human clinical use. Current in vivo and in vitro research has shed light on the effects of SO and its research has shown that SO can decrease low-density lipoprotein (LDL) levels while maintaining high-density lipoprotein (HDL) levels. Current limitations in recent studies include no standardized doses of SO given to subjects and unknown specific mechanisms of the different components of SO. Future studies should explore possible synergistic and adverse effects of SO when combined with current recommended pharmaceutical therapies and other adjunct treatments.

Categories: Cardiology, Family/General Practice, Public Health

Keywords: atherosclerosis, sesame oil, antioxidant, cardiovascular disease, anti-inflammatory

\section{Introduction And Background}

Atherosclerosis, the formation of plaques in arteries, has been the topic of extensive research due to its critical role in increasing the risk of cardiovascular diseases such as coronary heart disease (CHD) [1-2]. CHD is one of the leading causes of mortality and morbidity in Americans and causes approximately 610,000 deaths in the United States annually. Due to the high prevalence rate of one in four deaths due to CHD [1], many research studies have explored the mechanism of atherosclerotic plaque formation. Studies have come up with two prominent hallmarks of pathogenesis: the accumulation of cholesterol in the endothelial lining of arteries carried by low-density lipoproteins (LDLs) and chronic inflammation due to a high ratio of prooxidants to antioxidants [2-3]. Studies have implied that these hallmarks of atherosclerosis are not independent but are part of the same process where the abnormal deposition of LDLcholesterol (LDL-C) leads to an inflammatory response resulting in fatty plaques and vascular occlusion [4-5].

Previous literature has established that high serum cholesterol, more specifically elevated LDL levels, is a measure of atherosclerotic risk and, therefore, the reduction of plasma LDL levels 
should reduce the amount of atherosclerosis and its consequent risk of CHD [4]. LDL and highdensity lipoprotein (HDL) are carriers of cholesterol. LDL carries cholesterol to tissues in contrast to HDL, which carries cholesterol to the liver for disposal [6]. An increase of LDL in blood plasma can cause an imbalance in lipoproteins that can lead to build-up of cholesterol, especially in the tunica intima of the large and medium-sized arteries [7]. Cells of the vascular wall secrete oxidative products and can initiate lipid oxidation of the LDL. While the oxidized LDL levels increase and accumulate in the artery, damaged endothelial cells release inflammatory signals to initiate an immune response to fight the cholesterol aggregation $[2,8]$. This induces oxidative stress, an imbalance between pro-oxidants and antioxidants, and can cause oxidative damage to surrounding endothelial cells [3, 9]. Macrophages sense the signals and infiltrate the tunica intima to phagocytose the oxidized LDL aggregation [10-12]. With a chronic inflammatory response and the accumulation of oxidized LDL, a fatty streak in the arterial tunica intima forms [13]. Over time, the fatty streak grows, thickens the arterial walls, and contributes to chronic inflammation [14]. This leads to the development of a stable plaque, which can be at risk for eruption when it becomes unstable [15].

The guidelines for cholesterol management through the American College of Cardiology/American Heart Association recommend using statin therapy as the primary prevention treatment for managing cholesterol levels [16]. Statins are hydroxy-methylglutaryl coenzyme A (HMG-CoA) reductase inhibitors, which inhibit the HMG-CoA reductase, an essential rate limiting enzyme in the cholesterol synthesis pathway [17]. Previous literature has shown that statins are a powerful treatment option, which at a dose of $80 \mathrm{mg} /$ day can reduce LDL-C by about $47 \%$ [18]. The use of statins became more frequent when the Scandinavian Simvastatin Survival Study showed that long-term treatment with simvastatin was safe and improved survival rates in CHD patients [17].

While statin use has continued to grow in popularity, an issue with the statin treatment is the intolerance of the side effects associated with taking the drug [19]. Statin therapy is not for every patient; patients with higher levels of LDL-C are more likely to benefit from statin use than patients with lower levels of LDL-C [20]. About $10 \%$ of patients complain about the side effects, such as myalgia, and this decreases their patient compliance [16]. Due to the side effects of statins and other pharmaceutical drugs, the idea of using dietary and nutritional supplementation has gained traction in the scientific community [16]. The benefits of a dietary health regime have piqued curiosity because many different cultures have reaped health benefits through the ingredients in their cooking. For example, the use of sesame oil (SO) in Asian cultures has inspired studies on the dietary benefits of consuming SO. One study found that traditional Korean cooking called bugak prepared with pan-fried unroasted SO has been shown to decrease LDL, triglyceride (TG), and total cholesterol (TC) levels [21]. Experimental studies on rats fed a sesame seed extract exhibited a significant decrease in plasma cholesterol, triglycerides, very low-density lipoprotein (VLDL) cholesterol, and LDL. One study tested the effect of sesamin, a lignan in SO, in humans and found a significant reduction in LDL-C [22]. This study also referenced other literature, stating that sesamin may potentially reduce HMGCoA reductase activity by altering the amount of cholesterol ester and free cholesterol by decreasing acyl-CoA cholesterol acyltransferase (ACAT) activity [22]. By reducing HMG-CoA reductase activity, sesamin could potentially reduce LDL levels in a similar manner as statin drugs without the myopathy side effects.

While these studies were conducted many years ago, more recent studies into the properties of sesamin have concluded that sesamin and other constituents of SO have antihyperlipidemic effects and can improve many of the biochemical measurements found in a lipid panel. An experiment conducted on rats studied how sesamin influenced lipid metabolism because of the relationship between increased secretions of LDL in rat liver, following a decrease in fatty acid oxidation. This study concluded that sesamin appears to be a potent inducer of hepatic fatty acid oxidation and is an inhibitor of hepatic lipogenic enzyme gene expression by down 
regulating sterol regulatory element bind protein-1 (SREBP-1), which is a transcriptional factor that regulates gene expression for both fatty acid and cholesterol synthesis [23]. A more recent study extracted SO from Sesamum indicum L. and examined the effects on rabbits fed on a high fat diet (HFD). The study found that the rabbits supplemented with SO had a lower circulating level of LDL [24]. Sesamol is another lignan of SO that has also shown anti-inflammatory and antioxidant properties in studies [25].

All of these previous studies strongly indicate that SO supplementation has anti-atherogenic effects [26]. Despite indications of cholesterol-lowering effects through different constituents of sesame seeds and oil in many animal studies, the potential hypocholesterolemia properties of raw SO have not been tested much, to the best of our knowledge, in human studies.

The purpose of this literary review is to provide a broad overview of the effects of SO on atherosclerosis and its direction toward human clinical use. This review will examine the recent experimental studies that have been specifically conducted on SO and its effects on cholesterol levels and inflammation in animal atherosclerotic models and in vitro interactions between leukocytes involved in atherosclerosis and arterial tissues. The basis for this review is to select scientific research that tests SO or its lignans as a dependent variable in experiments about atherosclerosis and its hallmarks, inflammation or cholesterol. The time frame for research reviewed is from January 2010 to January 2016 in order to distinguish the most recent studies about SO.

\section{Materials and methods}

To begin the review article search, search words [SO] and [cholesterol or inflammation or atherosclerosis] were used to begin the search for papers that studied the effects of SO on levels of cholesterol or inflammation or atherosclerosis. The following criteria were used to further narrow down papers published:

\section{Published between January 2010 and January 2016.}

2. In vivo mammalian animal or human studies or in vitro studies.

3. Tests the use of SO, by itself or combined with other oils.

\section{Written in English.}

5. Topic pertains to SO's effects on atherosclerosis, cholesterol build-up, or inflammation.

\section{Results}

After the initial search of [SO] and [cholesterol, inflammation, or atherosclerosis] in the PubMed database, a total of 134 papers that included the key terms were found. After narrowing down the results with a time frame from January 2010 to January 2016, the number of studies dwindled down to 43 . Afterward, each abstract of the 43 search results were individually scanned with the criteria stated in the methods. The final search yielded 14 studies that experimented on the effects of SO on atherosclerotic risk factors, such as cholesterol levels and inflammation, and key players that initiate or lead to the progression of atherosclerosis. Below is a table of summary of each of the chosen studies (Table 1). 


\section{Cureus}

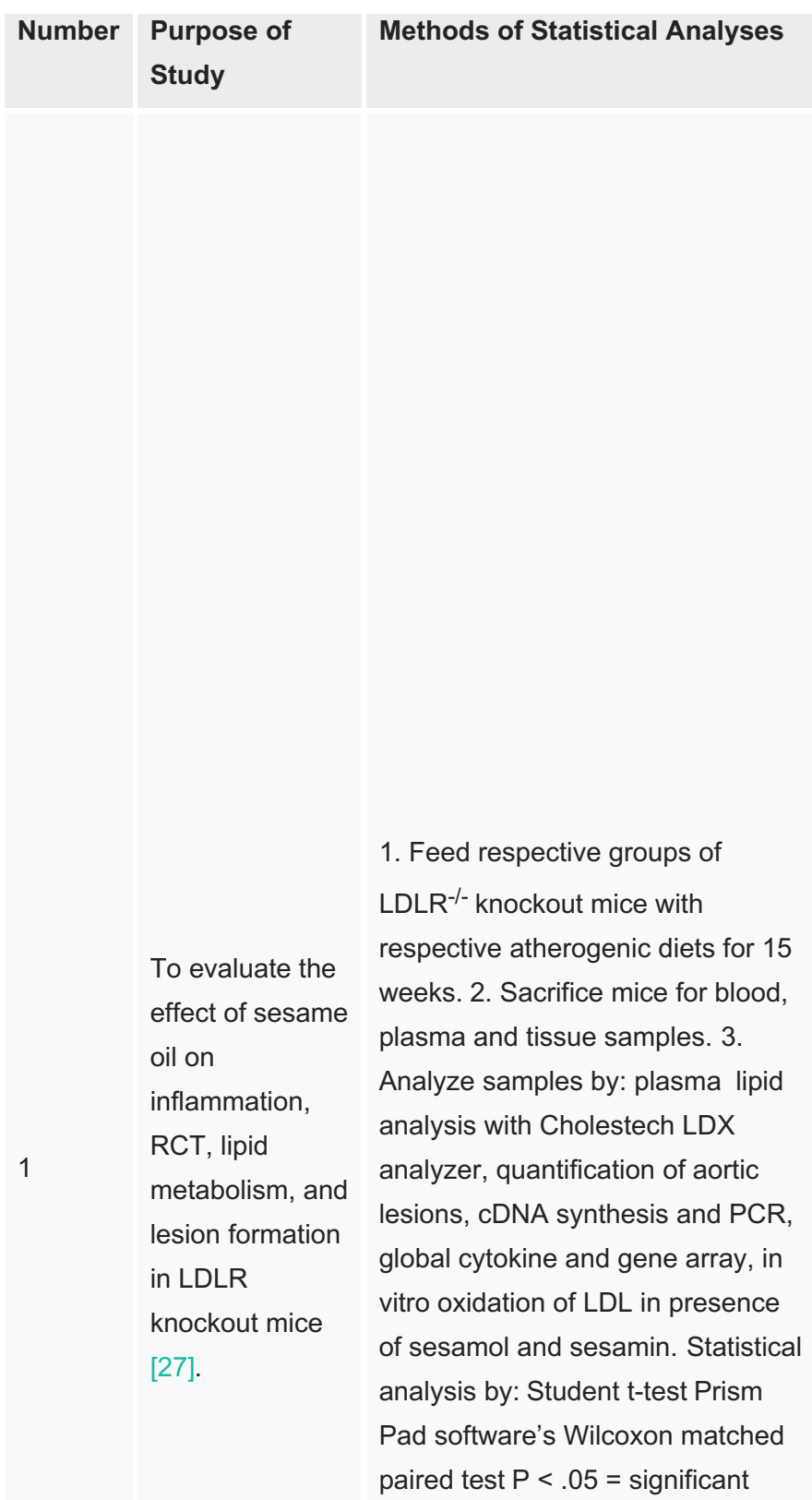

66 four-week-old

female LDLR $^{-/}$-

knockout mice that

weigh between $18 \mathrm{~g}$

and $20 \mathrm{~g}$ from

Jackson Laboratory.

Control: LDLR ${ }^{-/-}$

knockout female

mice fed an

atherogenic diet that

had $17 \%$ saturated

fat in milk.

Experimental: LDLR

I- knockout female

mice fed an

atherogenic diet that

replaced $17 \%$ of

saturated milk fat

with sesame oil.
Measured
[parameters]

Results

Percentage change

in weight plasma

lipid analysis TRG

TC LDL VLDL HDL

Quantification of

aortic lesions by

measuring surface

area. Gene array in

mice livers for

lipoprotein signaling

and cholesterol

metabolism. Gene

array in mice aorta

for mRNA

expression.

Cytokines array.

Insignifican

weight change

Plasma lipid

Analysis TRG: C

$>$ Exp TC: C >

Exp LDL: C >

Exp VLDL: C >

Exp HDL: $\mathrm{C}<$

Exp 30-40\%

reduction in Exp

than $\mathrm{C}$.

Quantification of aortic lesions $\mathrm{C}$

$>$ Exp in lesion size Gene array

in mice livers

showed

increased

expression of

genes related to

RCT and lipid

metabolism in

so fed mice.

Gene

expression in

mice aortas

showed that SO

fed mice had

increased

mRNA levels of the RCT gene

ABCA1, but

reduced levels

of $A B C G$,

monocyte

markers, and

scavenger

receptors.

Cytokines array

showed that

sesame oil

increased

expression of

genes pertaining

to RCT and

cholesterol

metabolism.

PCR array 


\section{Cureus}

1. For LDL oxidation test, oxidizing agents were added to extracted lipoproteins in presence and absence of SOAE. 2. For PCR array, macrophages pretreated with

[28] Selvarajan

K, et al. 2015

Apr To

evaluate the

effect of

Sesame Oil

Aqueous Extract

[SOAE] on

inhibiting

inflammation

and regulating

lipid metabolism
SOAE were incubated with LPS for

24 hours. Analysis by Qiagen array for atherosclerosis. 3. For real-time PCR analysis for inflammatory markers, HUVECS and macrophages were treated with SOAE and LPS/TNF-a. 4. Medium from macrophages resulting from SOAE and LPS treatments was collected and analyzed with ELISA 5. Webster mice were injected with SOAE and LPS intraperitoneally at varying concentrations. 6 . After two hours, mice were sacrificed and plasma and tissue were extracted. Plasma and tissues were analyzed. Statistical Analysis by: Student ttest $\mathrm{P}<.05=$ significant 8-week Swiss

Webster mice, RAW

264.7 cells, human

umbilical vein

endothelial cells

[HUVEC] Control:

Cells and mice not treated with sesame oil. Experimental:

Cells and mice treated with aqueous component of sesame oil.
1. Co-transfect Chinese hamster ovary cells with respective luciferase constructs with $\mathrm{pCMV-B-}$ galactosidase expression vector. 2 . After 24 hours of post-transfection, the transfectants were treated with different concentrations of sesamol showed that

SOAE reduced

LPS-induced

inflammation in

RAW 264.7

macrophage

cells. ELISA

showed

significantly

reduced

PCR array analysis:

Genes upregulated

expressions of

or downregulated

between

LPS+SOAE, LPS,

and SOAE

Cytokines analysis

ELISA for IL-6 and

TNF- $\alpha$ Measure of

NF-kB transcription

and translocation by

PCR array

IL-6 and TNF- $\alpha$

in macrophages and endothelial

cells in a

concentration-

dependent

manner. SOAE

was effective in

inhibiting LPS-

induced TNF- $\alpha$ and IL-6 levels

immunofluorescence in vivo at

Oxidation of

different

lipoproteins

measured in

absorbance over

time. Transport gene

expression analysis

by luciferase and

GFP activity.

concentrations, transcription and translocation of $\mathrm{NF}-\mathrm{kB}$, and oxidation of lipoproteins in vitro. SOAE activates liver $\mathrm{X}$ receptors, which regulate scavenger receptors expression and increased ATPbinding cassette A1 [ABCA1] mRNA

expression.

Sesamol and sesame oil upregulate PPARg1and

LXRa

expression $\sim 1.7$

[1] Watanabe heritable hyperlipidemic fold compared to

control $100 \mathrm{uM}$

Sesamol and 


\section{Cureus}

[29]

et al. 2015 Aug

To show the

anti-atherogenic

effects of:

1. sesamol on

Watanabe

heritable

hyperlipidemic

rabbits 2 .

sesame oil on

$\mathrm{LDLR}^{-/}$-mice by

the sesamol

403] and

sesame oil in

Watanabe

heritable

hyperlipidemic

rabbits and

LDLR-/- mice
Majdalawieh AF,

derivative [INV-

and sesame oil, with DMSO as a

negative control. 3. To test whether

MAPK pathway is affected by

sesamol and sesame oil, using the

same preparation stated in 1, 100

uM sesamol and $10 \mathrm{lg} / \mathrm{ml}$ sesame

oil were added to their respective

macrophages in the presence or

absence of $10 \mathrm{uM}$ of a MAPK

inhibitor. 4. To test whether

macrophage efflux improves with

sesamol and sesame oil treatment,

macrophages were treated with

different varying concentrations of

sesamol and sesame oil for 24

hours. Then they were induced to

uptake cholesterol. Amount of

cholesterol efflux was recorded 5 .

To test whether MAPK signaling

pathway is involved in the effects of

sesamol and sesame oil on

PPARg1 and LXRa transcriptional

activity, macrophages were treated

with DMSO, sesamol, or sesame

oil and MAPk inhibitor. Then they

were induced to uptake cholesterol.

Amount of cholesterol efflux was

recorded Statistical Analysis

Student unpaired T-test. $P<0.05$,

$P<0.01$, and $P<0.001$ are

significant.

1. Hypertensive male volunteers

must fast 12 hours from foods,

cigarettes, and medication, and 24

hours from liquids. Blood was

collected and an ultrasound was

used to determine endothelial

function. Patients were given their

oil [control or sesame] and

directions on ingesting it. 2 .

[30] Karatzi K, et Patients were given bread to eat.

al. 2013 Apr. To Two hours after ingestion, patient

examine the

possible effects

of sesame oil

4

consumption on

endothelial

function,

blood samples and ultrasound was

done again. 3. Patients enrolled in

the chronic phase of the study were

assigned to ingest sesame oil as

salad dressing for 60 days. At

systematic time intervals of the rabbits [2] $\mathrm{LDLR}^{-/-}$

mice [3] C57/BL6

mice, chow fed for 8-

12 weeks on chow-

diet, injected with $4 \%$

thioglycolate broth

solution

intraperitoneally.

Killed five days later

to collect peritoneal

macrophages [4]

Chinese hamster

ovary [CHO] cells,

transfected at 60-

$80 \%$ confluency for

luciferase reporter

assays and plasmid

DNA Control: Co-

transfected

peritoneal

macrophages treated

with DMSO

Experimental: Co-

transfected

peritoneal

macrophages treated

with sesamol or

sesame oil

Hypertensive males between 40-65 yr old. Hypertensive males had mean day blood systolic blood pressure $>135 \mathrm{mmHg}$ and diastolic blood pressure $>85 \mathrm{mmHg}]$ and were receiving antihypertensive medication. Control:
Tests effects of

sesamol and

sesame oil on

regulation of

transcriptional

activity and

expression PPARg1

and LXRa genes.

Measured by

transcriptional

activity and

expression in

luciverase reporter

and B-galactosidase

assays Tests

effects of sesamol

and sesame oil on

cholesterol efflux

Measured

cholesterol efflux by

$\mathrm{H}$-cholesterol efflux

assay.

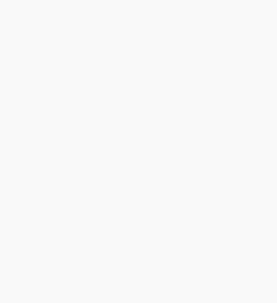

$10 \mathrm{ug} / \mathrm{ml}$ sesame

oil enhance

PPARc1 and

LXRa

transcriptional

activity in a

MAPK-

dependent

manner

Sesamol and

sesame oil

enhance

PPARc1 and

LXRa

transcriptional

activity in a

temporal

fashion, and this

enhancement is

statistically

significant at all

tested time

points except for

the $6 \mathrm{~h}$

treatment

Sesamol and

sesame oil

augment

macrophage

cholesterol efflux

in a MAPK-

dependent

manner

Postprandial and

long-term effects

of sesame oil

increased FMD

Postprandial effects

of sesame oil

Measured by flow-

mediated dilation

[FMD] and ICAM

Long-term effects of

sesame oil on FMD from baseline

comparison

ICAM decreased significantly at

60 days in the

long term study. 


\section{Cureus}

postprandial and study, Statistical Analysis by SPSS

long term intake. 18 for Windows Kolmogorov-

Smirnov test and Q-Q plots -

Tested all continuous variables for normal distribution ANCOVA Twosamples and paired samples student's t-tests - differences in measured variables between specific time points were evaluated by grouped comparisons $\mathrm{P}<0.017$ = significant

[31] Abdel-Daim MM, et al. 2016

Jan To

evaluate the protective and antioxidant potential of sesame oil [SO] and/ or a-lipoic acid [ALA] against DZN toxicity in male Wistar albino rats

[32] Korou LM, et al. 2014 Mar

To determine whether ingesting NAC and sesame oil beneficially induces hypolipidemic and antioxidant effects in a hyperlipidemic
1. Male Wistar rats were allowed to acclimate for two weeks before experimentation 2. Rats were injected with their respective fluids.

3. After one hour, all groups of rats were injected with DZN. 4. After four weeks, the rats were sacrificed and samples were collected from the blood, liver, kidney, and heart. 5. Analysis of samples by lipid profile. Statistical Analysis by GraphPad Prism statistical package version 5.0 for Windows ANOVA Tukey's multiple range tests $\mathrm{P} \leq$ $0.05=$ significant
1. Mice were fed one of four diets depending on what experimental group they belonged to. 2. After eight weeks, plasma, blood, aortic and hepatic tissues were collected. 3. To assess data: - a lipid profile for plasma - measurement kits for peroxide and NO amount -

hematoxylin-eosin staining of aorta and liver Statistical Analysis by: 2way ANOVA - Measured body weight, food consumption, serum lipid, total peroxides, NO and hepatic enzymes levels with diet and NAC intake or diet and sesame oil ANCOVA - compare T1 measurements after controlling for baseline levels. 1-way ANOVA Hypothalamic GR protein levels and plasma corticosterone levels
Hypertensive men on and ICAM-1

control oil

Experimental:

Hypertensive men on

sesame oil
ICAM did not decrease significantly for the short term postprandial
Male Wistar rats,

weighing 160-200g,

and allowed to

acclimate before

experiment. Control:

Saline or DZN

injected rats

Experimental: SO,

ALA, or SO and ALA injected mice. 12-week-old male

C57bl/6 mice

Control: [NC] C57bl/6 mice on basal diet

Experimental: $[\mathrm{HC}]$ C57bl/6 mice on high cholesterol diet [ $2 \%$ cholesterol and $0.5 \%$ cholic acid ]for eight weeks $[\mathrm{HCN}]$ C57bl/6 mice on high cholesterol diet with NAC

supplementation [230 mg/kg p.o.] [HCS]-HCS
Lipid profile

compared to DZN

TC LDL-C HDL-C TCG

SO and/or ALA

supplementation ameliorated the deleterious effects of DZN intoxication.

Higher serum levels of total and LDL-

cholesterol were recorded in all groups fed the high cholesterol diet. HCN group showed lower lipid levels compared to $\mathrm{HC}$ and HCS groups, where there was no

Lipid profile TC LDL- observed C HDL-C TCG difference

Total Serum between HCS

concentrations of: Peroxides NO Hematoxylin and eosin staining of aortic and hepatic tissues and $\mathrm{HC}$ groups. $\mathrm{HCN}$ and HCS groups had a significant decrease of lipid peroxidation compared to 


\section{Cureus}

murine model

Kruskall-Wallis test Mann-

Whitney's U test - Histological

scores Benjamini and Hochber's

False Discovery Rate - Assess

differences between multiple

groups, as well as to control family-

wise error to $\mathrm{a}, 0.05 \mathrm{P} \leq 0.05=$

significant
[33] Sharma AK, 1. Rats were fed with HFD [55\%

et al. 2012 Nov. calorie from fat and $2 \%$ cholesterol]

To determine

the role of

sesamol in

chronic high-

cholesterol/high-

fat diet [HFD]-

induced CMetS

in rats. for 60 days. 2 . On the 30th day, rats were administered sesamol 2,4 and $8 \mathrm{mg} / \mathrm{kg}$ per day for the next 30 days. Statistical Analysis by SPSS software package version 11.5. 1 way ANOVA Bonferroni post hoc test. $\mathrm{P}<.05=$ significant.

\section{C57bl/6 mice fed}

high cholesterol diet

enriched with $10 \%$

sesame oil for 8

weeks

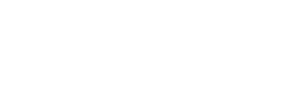

\section{Male Wistar albino} rats weighing 140 -

$170 \mathrm{~g}$ and are 6-8

weeks old. Control:

Rats fed with regular chow Rats fed with high fat chow diet

Experimental: Rats fed with pioglitazone $10 \mathrm{mg} / \mathrm{kg}$ per day, and rats on high fat chow diet and varying amounts of sesamol.

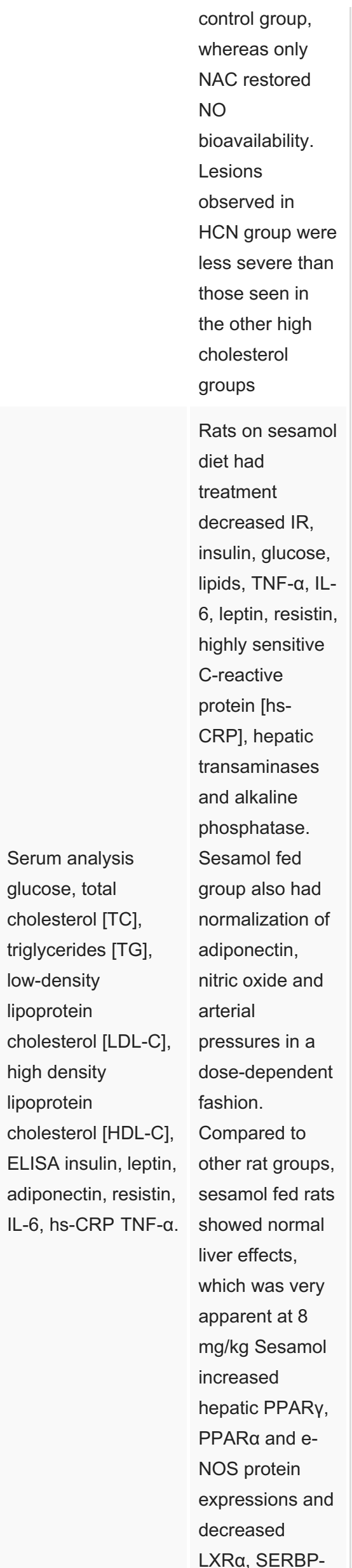

control group,

NAC restored

$\mathrm{NO}$

Lesions

observed in

$\mathrm{HCN}$ group were

less severe than

those seen in

Rats on sesamol

diet had

treatment

decreased IR

insulin, glucose,

lipids, TNF- $\alpha$, IL-

leptin, resistin

C-reactive

protein [hs-

CRP], hepatic

transaminase

and alkaline

phosphatase.

group also had

normalization of

LXRa, SERBP- 


\section{Cureus}

[24] Asgary S, et al. 2013 Sept.

To evaluate the

effects of

sesame seed and sesame oil on serum lipids, apolipoproteins, liver enzymes, glucose, and insulin in a hyperlipidemic rabbit model
1. Allow rabbits to acclimate for two weeks 2 . Feed rabbits on their respective diets for eight weeks. 3 . Analyze serum cholesterol levels. Statistical Analysis by SPSS software version 13.0 KruskalWallis test. Dunn's Test $\mathrm{P}<0.05=$ significant

Adult male rabbits of

New Zealand strain

[1.25-2.50kg]

Control: Rabbits on normal diet or

hypercholesterolemic diet Experimental:

Rabbits on

hypercholesterolemic

Serum cholesterol

TC HDL-C LDL-

\section{$\mathrm{C}+\mathrm{VLDL}-\mathrm{C}$}

diet with $10 \%$

sesame seeds or

hypercholesterolemic diet with $5 \%$ sesame oil

1. Rats were placed on a high fat diet with respective oil diets with single or blended oils. The diet

[34] Reena MB, consists of $10 \%$ fat from native oils; et al. 2011 Jan. coconut oil [CNO], rice bran oil To study the [RBO], or sesame oil [SESO]; effect of blended and inter esterified oils

blended [B]; $\mathrm{CNO}+\mathrm{RBO}[\mathrm{B}]$ or $\mathrm{CNO}+\mathrm{SESO}[\mathrm{B}]$ and inter esterified oil [I]; CNO+RBO[I] or CNO+SESO[I] for 60 days 2 . Transcriptional profiling of genes acid compositions on gene expression involved in

cholesterol homeostasis in rat liver. involved in cholesterol homeostasis was studied after feeding rats with a semi purified diet Statistical analysis by SPSS statistical software 1-way ANOVA followed by Duncan's multiple range test. Pearson correlation coefficients were calculated. $P \leq 0.05=$ significant. 1c, P-JNK and

$\mathrm{NF}-\mathrm{kB}$

expression.

Supplementation with sesame oil, but not sesame seed, can ameliorate serum levels of lipids and hepatic enzymes in rabbits under a high-fat diet

Hepatic LDL receptor [LDLR] expression significantly increased in rats fed inter esterified oils by 100-200\% compared with rats fed blended oils and by 400 $500 \%$ compared with rats fed CNO. Positional alteration in fatty acids of oils used in the diet induced

changes in

LDLR

expression, which was accompanied by parallel changes in cholesterol-

7a-hydroxylase [CYP7A1] and SREBP-2 genes

Sesamol fed hamsters in showed reduced plasma L5 levels 


\section{Cureus}

$$
\text { and }
$$

atherosclerosis development in Syrian hamsters and on the L5induced apoptosis of ECs.

[35] Chen WY, et al. 2015 Feb.

To study the effects of sesamol on plasma L5 levels

\section{To test effects of sesamol on} plasma L5, Syrian hamsters were fed respective atherosclerotic diets for 16 weeks. 2. Collected blood, plasma, and tissues 3. Treated endothelial cells with L5 to induce apoptosis 4. treated experimental group with sesamol Statistical Analysis by: Student's t-test. $\mathrm{P}<$ $0.05=$ significant
Syrian hamsters, and

Human aortic

endothelial cells

Control: Syrian

hamsters that are fed

a normal diet. Syrian

hamsters fed a high

fat diet Human aortic

endothelial cells that

were not treated with

plasma L5

Experimental: Syrian hamster that are fed a high fat diet with sesame oil. Human aortic endothelial cells that were treated with L5
Gel electrophoresis to determine electronegativity of L5 Liquid chromatography of L5 plasma levels Lipid profile Observations of Oil Red O staining to count lesions Fluorescence observation and immunoblot analyses of activated caspase-3 to show apoptosis/ activity levels of LOX-1, MAPK, and eNOS. when compared

with the HFD

group. Sesamol

fed hamsters

showed smaller atherosclerotic

lesion size in the aortic arch compared with the HFD group. In human aortic ECs, 0.3-3 $\mu \mathrm{M}$ of sesamol blocked L5induced apoptosis in a dose-dependent manner. Cells treated with sesamol showed inhibition of the

L5-induced lectin-like oxidized LDL receptor-1 [LOX 1] - dependent phosphorylation of p38 MAPK and activation of caspase-3 and increased phosphorylation of eNOS and Akt.

Serum and liver lipids showed significant decrease in Total cholesterol [TC],

Triglyceride

[TG], LDL-C

Male Wistar rats [OUTB-Wistar, IND-cft [2c]] weighing $62 \pm 5 \mathrm{~g}$, Control: Rats fed one type of oil in their diet Experimental: Rats fed a blended oil in levels in GCO

and GCO

Lipid analysis TC blended oil fed LDL-C HDL-C ALA EPA DHA

Arachidonic Acid rats compared to native oil fed rats. ALA, EPA, DHA levels increased while 


\section{Cureus}

modulatory

effect on lipid

metabolism. their diet.

significant

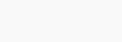

Plasma lipid profile TC LDL VLDL HDL TG FFA PL

Myocardial and

Hepatic Tissue Lipids TC TG FFA PL Histopathological examination of liver tissues rats $\mathrm{mg} / \mathrm{kg} \mathrm{BW]}$

\section{of body weight] subcutaneously for
two days to induce myocardial
infarction 2 . Administer sesamol
dissolved in $0.9 \%$ saline for seven
days 3. Tissue collection on
ninth day. Collected blood, plasma,
heart, and liver. Statistical Analysis
by: ANOVA Duncan's Multiple
Range Test [DMRT] using SPSS
Software Package v.10.0. P $\leq 0.05$ \\ = significant \\ 1. Injecting isoproterenol $85 \mathrm{mg} / \mathrm{kg}$
of body weight] subcutaneously for
two days to induce myocardial
infarction 2 . Administer sesamol
dissolved in $0.9 \%$ saline for seven
days 3 . Tissue collection on
ninth day. Collected blood, plasma,
heart, and liver. Statistical Analysis
by: ANOVA Duncan's Multiple
Range Test [DMRT] using SPSS
Software Package v.10.0. P $\leq 0.05$ \\ 1. Injecting isoproterenol $85 \mathrm{mg} / \mathrm{kg}$
of body weight] subcutaneously for
two days to induce myocardial
infarction 2 . Administer sesamol
dissolved in $0.9 \%$ saline for seven
days 3 . Tissue collection on
ninth day. Collected blood, plasma,
heart, and liver. Statistical Analysis
by: ANOVA Duncan's Multiple
Range Test [DMRT] using SPSS
Software Package v.10.0. P $\leq 0.05$ \\ 1. Injecting isoproterenol $85 \mathrm{mg} / \mathrm{kg}$
of body weight] subcutaneously for
two days to induce myocardial
infarction 2 . Administer sesamol
dissolved in $0.9 \%$ saline for seven
days 3 . Tissue collection on
ninth day. Collected blood, plasma,
heart, and liver. Statistical Analysis
by: ANOVA Duncan's Multiple
Range Test [DMRT] using SPSS
Software Package v.10.0. P $\leq 0.05$}

.

linoleic acid

[LA], arachidonic acid [AA] levels decreased in different tissues of GCO and GCO blended oils fed rats

Sesamol almost normalized serum ISOadministered effects [elevated of TC, LDL-C,

VLDL-C and a reduced of HDL$\mathrm{C}]$ and reduced plasma TG, FFA and $\mathrm{PL}$

significantly. ISO-

administered rats showed significant increase in tissue TC, TG and FFA with a reduction in $\mathrm{PL}$. Improvement was observed in the levels of these

parameters to near normality in rats treated with sesamol. ISOinduced rat group showed dilated central vein surrounded by necrotic hepatocytes. Sesamol [50 $\mathrm{mg} / \mathrm{kg} \mathrm{BW]}$ treatment showed the central vein surrounded by normal hepatocytes with mild necrosis. 


\section{Cureus}

[38] $\mathrm{Wu} \mathrm{XL,} \mathrm{et}$ al. 2015 Aug.

To test whether sesamol has anti-

inflammatory activity in NF-kB and MAPK pathways in LPS-stimulated macrophages
1. RAW 264.7 macrophages were treated with sesamol, then LPS. 2. Pro-inflammatory cytokines were analyzed with ELISA 3. COX-2 and iNOS was analyzed by PCR 4. Nrf2 was analyzed by Western blots Statistical Analysis by: ANOVA Post hoc analyses with Dunnett's test $P<0.05=$ significant
1. Sesame and sunflower oil mix

[39] Sudhaker B, given to 4 groups of patients for 45 et al. 2011 Feb.

To compare the effects of

sesame oil and sunflower oil mix on patients taking Nifedipine. days. 2. Measured blood pressure

lipid peroxidative markers, enzymatic and non-enzymatic antioxidants, lipid profiles and electrolytes in blood. Statistical Analysis by ANOVA Tukey's multiple range tests on GraphPad Prism statical package ver. 5.0 P $\leq$ $0.05=$ significant

\section{RAW 264.7}

macrophages from BCRC, Taiwan.

Control: RAW 264.7 macrophages that were not treated with sesamol

Experimental: RAW 264.7 macrophages that were treated with sesamol
Sesamol

inhibited

production of

Viability assay NO

nitric oxide,

production ELISA

prostaglandin E2

to measure

cytokines,

chemokines, and

[PGE2], and

proinflammatory

PGE2 @ 450 nm

cytokines.

to measure COX-2, mRNA and

$\mathrm{HO}-1$, iNOS, Nrf2, protein

IkB-a, Lamin B1, expression of

Phosphorylated-ikb- iNOS and COX-

a, p65, adenosine

2 , and enhanced

monophosphate-

activated protein

kinase [AMPK],

antioxidant

pathway

ERK1/2, p38, INK, phosphorylated-

represented by

Nrf2 and HO-1.

AMPK Real time

Sesamol

suppressed NF-

PCR to evaluate

$\mathrm{kB}$ transport and

gene expression

decreased

Production of ROS

MAPK

activation, but it

increased AMPK

activation.

Blood Pressure

[mmHg] Lipid Profile

TC [mg/dL] TGC

[mg/dL] HDL

$[\mathrm{mg} / \mathrm{dL}]$ VLDL

$[\mathrm{mg} / \mathrm{dL}] \mathrm{LDL}[\mathrm{mg} / \mathrm{dL}]$

14 normal male

patients and 38 male

patients [45-55 yrs

old] with mild to

moderate

hypertension [SBP

$>140 \mathrm{mmHG}$ or DBP

$<90 \mathrm{mmHG}$ ] on

treatment for

nifedipine. Control:

Normal patients

Hypertensive

patients on nifedipine

Experimental:

Hypertensive

patients on nifedipine

and

sesame+sunflower
TC/HDL Antioxidant

status Superoxide

dismutase $\left[\mathrm{U}^{*} / \mathrm{mg}\right.$

$\mathrm{Hb}$ ] Catalase [U*/mg

$\mathrm{Hb}$ ] Glutathione

peroxidase $\left[U^{*} / \mathrm{mg}\right.$

$\mathrm{Hb}$ ] Vitamin E

[mg/dL] Vitamin C

[mg/dL] Beta-

carotene $[\mathrm{mg} / \mathrm{dL}]$

Reduced glutathione

[mg/dL] Lipid

peroxidative

acid reactive

substances

[nmol/mL] markers Barbituric
Nifedipine and

oil-mix patients

showed a

significant

decrease in

blood pressure, lipid peroxidative markers, lipid profile [excludes HDL levels], sodium, and chloride in comparison to nifedipine only patients.

Nifedipine and oil-mix patients showed a significant increase in enzymatic antioxidants, non-enzymatic 


\section{Cureus}

oil mix.

Conjugated dienes

antioxidants,

[nmol/mL] Sodium

$\mathrm{HDL}$, and

[mEq/L] Potassium

potassium levels

[mEq/L] Chloride

in comparison to

[mEq/L]

nifedipine only

patients.

\section{TABLE 1: Table of Results}

AA: Arachidonic Acid; ABCA1: ATP-binding Cassette A1; ALA: a-linolenic acid; AMBK: Adenosine Monophosphate-activated Protein Kinase; ANCOVA: Analysis of Covariance; ANOVA: Analysis of Variance; CHO: Chinese Hamster Ovary; DMRT: Duncan's Multiple Range Test; DMSO: Dimethyl Sulfoxide; ELISA: Enzyme-linked Immunosorbent Assay; FMD: Flow-mediated Dilation; GCO: Garden Cress Oil; HDL: High density Lipoprotein; HDL-C: HDL- Cholesterol; HFD: High-fat Diet; hs-CRP: Highly Sensitive C-Reactive Protein; HUVEC: Human Umbilical Vein Endothelial Cell; ICAM-1: Intercellular Adhesion Molecule 1; LA: Linoleic Acid; LDL: Low-density Lipoprotein; LDL-C: LDL-Cholesterol; LDL-C: low-density Lipoprotein Cholesterol; LDLR: LDL receptor; LDLR: Low-density Lipoprotein Receptor; LPS: Lipopolysaccharide; PCR: Polymerase Chain Reaction; PGE2: Prostaglandin E2; RCT: Reverse Cholesterol Transport; SOAE: Sesame Oil Aqueous Extract; TC: Total Cholesterol; TG: Triglyceride; VLDL: Very Low-density Lipoprotein. SPSS Software Package v.10.0. (IBM, NY, USA.)

Out of the 14 studies in Table 1, studies 4 and 14 were the only two studies using human subjects. Study 4 studied SO and its effects on endothelial function in hypertensive men. The results were that postprandial and long-term effects of SO increased flow-mediated dilation (FMD) from baseline comparison. The measured levels of intracellular adhesion molecules (ICAM) decreased significantly at 60 days in the long-term study but did not decrease significantly in the short-term postprandial study. The results of this study showed that SO has both local and short acting benefits such as vasodilation and also longer acting properties involved with the downregulation of the integrin ligand of the ICAM. Since the ICAM did not decrease significantly for the postprandial two-hour period, but did for the long-term period, this may suggest that the downregulation of the ICAM protein is due to the SO changing the gene expression of the ICAM gene. Study 14 was the second study out of the 14 studies that had human participants. The study was comparing nifedipine against nifedipine and an oil mix of SO and sunflower oil. Nifedipine and oil-mix patients showed a significant decrease in blood pressure, lipid peroxidative markers, lipid profile (excludes HDL levels), sodium, and chloride in comparison to nifedipine-only patients. Nifedipine and oil-mix patients showed a significant increase in enzymatic antioxidants, non-enzymatic antioxidants, HDL, and potassium levels in comparison to nifedipine-only patients.

The alteration of gene expression is supported by studies $1,2,3,7,9$, and 13, which all show that SO's mechanism of action includes the change in gene expression to inflammatory or lipid metabolism proteins. Study 1 found that mice livers showed increased expression of genes related to the reverse cholesterol transport (RCT) and lipid metabolism in SO-fed mice. Gene expression in mice aortas showed that SO-fed mice had increased mRNA levels of genes related to RCT but reduced levels of monocyte markers, ABCG, and scavenger receptors. Cytokines array showed that SO increased expression of genes pertaining to RCT and cholesterol metabolism. This indicates that SO is associated with increasing or decreasing of certain proteins. This study fed the mice the SO diet for 15 weeks, which is congruent with the idea of long-term gene expression changes. Study 1 is not the only study though that showed that SO could have short-term benefits also. Study 2 showed in mice not only that the expression of inflammatory mediators could be significantly reduced but also that the sesame oil aqueous extract (SOAE) could inhibit the oxidation of lipoproteins through lipopolysaccharides (LPS)induced tumor necrosis factor (TNF)- $\alpha$ and interleukin (IL)-6. Study 3 examined SO and its ability to augment macrophage cholesterol efflux through a mitogen-activated protein kinase (MAPK) signaling pathway. 
Study 5 examined $\alpha$-lipoic acid and SO's antioxidant protective property from diazinon (DZN) toxicity. The rats were sacrificed after four weeks, which showed that the combination of $\alpha$ lipoic acid and SO was able to ameliorate the DZN intoxication. Study 6 showed that SO significantly decreased lipid peroxidation but did not significantly increase nitric oxide compared to n-acetyl cysteine. Also, the results found that 10\% SO in the HFD for eight weeks in the mice did not decrease the lipid levels significantly compared to the control. The decrease in lipid peroxidation is consistent with the other studies that found that SO could affect lipid metabolism. Study 6 does not seem to be consistent with study 4 , which showed both short- and long-term FMD. FMD is due to the sheer stress stimulus that produces a nitric oxide-dependent response. FMD is a direct marker of nitric oxide (NO) bioavailability and study 6 showed that only NAC restored NO bioavailability and not sesame oil supplementation. Study 4 was conducted on hypertensive males, and study 6 was conducted on mice. Study 7 showed that sesamol could decrease lipids and increase hepatic protein expression, while study 8 found that SO and not sesame seed could ameliorate high lipid levels and hepatic enzymes in rabbits fed a HFD. Studies 9 to 12 supported increased hepatic enzymes to decrease lipid levels. Study 13 was consistent with study 6 and found that sesamol inhibited production of NO and other proinflammatory markers.

\section{Review}

In the studies that analyzed the effect of SO and its lignans on a lipid profile, SO has been shown to decrease TC, LDL, and VLDL plasma levels in hypercholesterolemic rodent and rabbit models. However, one reviewed study had stated that SO has no significant effects on lipid levels compared to the control [32]. Study 6 stated that SO treatment was not significantly different from the control treatment, which supported that the beneficial effects resulting from treatment with N-acetylcysteine (NAC) and SO together was only due to NAC; there was not enough SO to counteract the high cholesterol diet, which implies that SO treatment may be dose dependent. Other studies also agree that their research results that indicate the benefits of SO were truly significant at a certain dose [33-35]. Plant studies showed that plant stanols decreased LDL levels in a dose-dependent manner up to $~ 17 \%$ in a linear fashion when given up to 9 grams/milliliter [40]. The diet fed to the subjects in study 6 was composed of $10 \%$ SO compared to other studies that administered SO amounts depending on the weight of the subject. Depending on the weight of the subjects, $10 \%$ of SO in the diet may not have been enough to show SO's anti-lipidemic effects [41]. Since caloric intake is higher than recommended in many populations, research has pointed out that consumption of supplemental oil should be associated with reduced intake of saturated fat [42]. This implies that SO could also be present in high amounts but not enough to induce its effects relative to the subject ingesting it.

In order to research additive effects of SO, some research explored the effects of mixed oils containing SO. Many vegetable oils, like sunflower or olive oils, also show hypocholesterolemic effects when ingested [43]. Research on the use of SO to treat atherosclerosis has yet to accumulate studies and research. So far, there are mixed reviews about SO, some studies stating that SO is not as effective as other edible oils. Study 6 studied the effects of SO in comparison to other oils in order to compare SO as a novel nutritional element to other vegetable oils that are part of our daily nutrition. Results in the study showed that SO was not as effective as the other oils [30]. This can be due to a few reasons, one being that the SO is not isolated and tested on its own. Blending different oils could result in a synergistic, antagonistic, or neutral effect. SO on its own can decrease cholesterol levels in the blood, but studies showed that SO blended with $\alpha$-lipoic acids decreased cholesterol levels in the blood much more than $\alpha$-lipoic acids or SO alone [31]. Another reason could be that there is no universal SO used throughout all the studies. There are many brands of SO, which are made with different techniques and compositions that may influence the effects of SO [44]. For example, SO is derived from heating sesame seeds to a certain temperature in order to create many of the lignans that are abundant 
in the oil but not the seeds [45]. This may increase or decrease the potency of SO compared to other vegetable oils and vice versa. In order to be able to properly compare the studies, one standardized concoction of SO should be created and consistently used throughout the studies.

Another way research explores the effects of SO is to test it in a mix with other components. For example, study 14 compared the effects of nifedipine to the effects of an oil mix of nifedipine with SO and sunflower oil on hypertensive patients, which resulted in a decrease in hypertensive factors. However, it is possible that the benefits could have been from the sunflower oil alone or that the SO had no benefit. It is also possible that the SO could have had adverse effects that negated some of the benefits of nifedipine or vice versa. The conclusion is that many of these studies are limited because they do not isolate the benefit of SO in humans alone and that different concentrations of SO are used in different studies and that a future study should examine the different concentrations of SO and its effects on humans with hyperlipidemia, hypertension, and diabetes mellitus. New studies can compare patients treated with differing mixes of medication, such as nifedipine, statins, metformin, with different concentrations of SO. Like statin therapy, many studies have promising results that show SO, when used as a dietary supplement, to lower LDL levels in the blood in coronary and aortic vasculature, as well as the liver and the brain [46-47]. The 14 studies did not examine the effects of SO on pancreatic beta cells, and future studies may want to see if SO has any potential effects since diabetes is also often associated with increased cardiovascular disease.

In most of the mentioned studies performed on animal models with SO, the benefits of SO and its components (e.g., sesaminol) can change the amount of gene transcription in different targets, such as endothelial cells, hepatocytes, and macrophages. Lignans such as sesamin and sesamol have gained popularity as they have been shown to have antioxidant and hypocholesterolemic effects [48-49]. However, many more studies need to be conducted on human participants since only two out of the 14 were on humans and one of the studies did not examine SO directly. In study 4, hypertensive men who took SO showed an increase in shortand long-term FMD and long-term ICAM. While these results look promising, there is a lot more that needs to be examined. In contrast, study 14 compared the benefits of SO and nifedipine with nifedipine alone. Neither of these studies compared the efficacy of SO to current pharmaceutical treatments in treating atherosclerosis. Previous studies have looked at the synergistic benefits as opposed to a direct comparison of SO with insulin-independent diabetic medications and anti-hypertensive medications [50]. These results show that in order to properly assess the effects of SO, there is a strong requirement for human trials with SO. Animal studies have shown that SO has decreased atherosclerotic factors without significant harm to the models, but it is unknown whether the same effects of SO would affect humans in the same way. In order to one day implicate SO and its effects on atherosclerotic patients, SO must past more clinical trials in order to gain traction as a possible treatment for atherosclerosis and other cardiovascular diseases.

\section{Conclusions}

SO research shows promise in decreasing high levels of cholesterol and inflammation, lowering risks of atherosclerosis, and delaying the onset of cardiovascular diseases. Since SO is very inexpensive and natural, progressing research on SO to someday implement SO as a good pharmaceutical treatment would be an investment, especially when SO has yet to show adverse effects. However, SO has not had many clinical trials, and the benefits relative to other oils and medications still need to be investigated. This literature review found that the benefits of SO vary between studies due to the methodology of SO product, dose dependence, and examination of different variables. Many of these studies are limited because they do not isolate the benefit of SO in humans alone and because there are different concentrations of SO used in each study. Future studies should examine the different concentrations of SO and its effects on humans with hyperlipidemia, hypertension, and diabetes mellitus in a dose-dependent manner 
relative to the patient's body habitus. Future studies can also look at synergism by comparing patients treated with differing combinations of medication, such as nifedipine, statins, metformin, with different concentrations of SO relative to the individual's saturated fat diet.

\section{Additional Information \\ Disclosures}

Conflicts of interest: In compliance with the ICMJE uniform disclosure form, all authors declare the following: Payment/services info: All authors have declared that no financial support was received from any organization for the submitted work. Financial relationships: All authors have declared that they have no financial relationships at present or within the previous three years with any organizations that might have an interest in the submitted work. Other relationships: All authors have declared that there are no other relationships or activities that could appear to have influenced the submitted work.

\section{References}

1. Mozaffarian D, Benjamin EJ, Go AS, et al.: Heart disease and stroke statistics-2016 update: a report from the American heart association. Circulation. 2015, 133:16.

10.1161/CIR.0000000000000350

2. Ross R: The pathogenesis of atherosclerosis: a perspective for the 1990s . Nature. 1993, 362:801-809. 10.1038/362801a0

3. Dhalla NS, Temsah RM, Netticadan T: Role of oxidative stress in cardiovascular diseases . J Hypertens. 2000, 18:655-673.

4. Parthasarathy S, Steinberg D, Witztum JL: The role of oxidized low-density lipoproteins in the pathogenesis of atherosclerosis. Annu Rev Med. 1992, 43:219-225.

10.1146/annurev.me.43.020192.001251

5. Tedgui A, Mallat Z: Cytokines in atherosclerosis: pathogenic and regulatory pathways . Physiol Rev. 2006, 86:515-581. 10.1152/physrev.00024.2005

6. Alagona P Jr.: Beyond LDL cholesterol: the role of elevated triglycerides and low HDL cholesterol in residual CVD risk remaining after statin therapy. Am J Manag Care. 2009, 15:65-73.

7. Stary HC: Lipid and macrophage accumulations in arteries of children and the development of atherosclerosis. Am J Clin Nutr. 2000, 72:1297-1306.

8. Goligorsky MS: Endothelial cell dysfunction: can't live with it, how to live without it . Am J Physiol Renal Physiol. 2005, 288:871-880. 10.1152/ajprenal.00333.2004

9. Loffredo L, Zicari AM, Occasi F, et al.: Endothelial dysfunction and oxidative stress in children with sleep disordered breathing: role of NADPH oxidase. Atherosclerosis. 2015, 240:222-227. 10.1016/j.atherosclerosis.2015.03.024

10. Mosser DM, Edwards JP: Exploring the full spectrum of macrophage activation . Nat Rev Immunol. 2008, 8:958-969. 10.1038/nri2448

11. Ikonomidis I, Lekakis J, Vamvakou G, et al.: Cigarette smoking is associated with increased circulating proinflammatory and procoagulant markers in patients with chronic coronary artery disease: effects of aspirin treatment. Am Heart J. 2005, 149:832-839. 10.1016/j.ahj.2004.08.030

12. O'Byrne D, Devaraj S, Islam KN, et al.: Low-density lipoprotein (LDL)-induced monocyteendothelial cell adhesion, soluble cell adhesion molecules, and autoantibodies to oxidizedLDL in chronic renal failure patients on dialysis therapy. Metabolism. 2001, 50:207-215. 10.1053/meta.2001.19486

13. Svensson J, Sjogren K, Levin M, et al.: Increased diet-induced fatty streak formation in female mice with deficiency of liver-derived insulin-like growth factor-I. Endocrine. 2016, 52:550560. 10.1007/s12020-015-0809-1

14. Aloi M, Tromba L, Rizzo V, et al.: Aortic intima-media thickness as an early marker of atherosclerosis in children with inflammatory bowel disease. J Pediatr Gastroenterol Nutr. 2015, 61:41-46.

15. Ito H: Omega-3 polyunsaturated fatty acids and coronary unstable plaque. Hint to further 
reduce coronary events. Circ J. 2013, 77:2473-2474. 10.1253/circj.CJ-13-0851

16. Grundy SM: Statins for All?. Am J Cardiol. 2014, 11:1443-1446. 10.1016/j.amjcard.2014.08.032

17. Scandinavian Simvastatin Survival Study Group: Randomised trial of cholesterol lowering in 4444 patients with coronary heart disease: the Scandinavian Simvastatin Survival Study (4S). Lancet. 1994, 19:1383-1389. 10.1016/S0140-6736(94)90566-5

18. Pedersen TR, Tobert JA: Simvastatin: a review . Expert Opin Pharmacother. 2004, 5:25832596. 10.1517/14656566.5.12.2583

19. Kutner JS, Blatchford PJ, Taylor DH Jr., et al.: Safety and benefit of discontinuing statin therapy in the setting of advanced, life-limiting illness: a randomized clinical trial. JAMA Intern Med. 2015, 175:691-700. 10.1001/jamainternmed.2015.0289

20. Soran H, Schofield JD, Durrington PN: The importance of considering LDL cholesterol response as well as cardiovascular risk in deciding who can benefit from statin therapy. Curr Opin Lipidol. 2014, 25:239-246. 10.1097/MOL.0000000000000097

21. Kim M, Hong SH, Chung L, et al.: Superiority of traditional cooking process for bugak (Korean traditional fried dish) for plasma lipid reduction. J Med Food. 2014, 17:57-66. 10.1089/jmf.2013.3057

22. Hirata F, Fujita K, Ishikura Y, et al.: Hypocholesterolemic effect of sesame lignan in humans . Atherosclerosis. 1996, 26:135-136. 10.1016/0021-9150(95)05769-2

23. Ide T, Ashakumary L, Takahashi Y, et al.: Sesamin a sesame lignan, decreases fatty acid synthesis in rat liver accompanying the down-regulation of sterol regulatory element binding protein-1. Biochim Biophys Acta. 2001, 30:1-13. 10.1016/S1388-1981(01)00167-6

24. Asgary S, Rafieian-Kopaei M, Najafi S, et al.: Antihyperlipidemic effects of Sesamum indicum L. in rabbits fed a high-fat diet. ScientificWorldJournal. 2013, 2013:1-5. 10.1155/2013/365892

25. Wanasundara PK, Shahidi F: Process-induced changes in edible oils . Adv Exp Med Biol. 1998, 434:135-160. 10.1007/978-1-4899-1925-0 13

26. Bhaskaran S, Santanam N, Penumetcha M, et al.: Inhibition of atherosclerosis in low-density lipoprotein receptor-negative mice by sesame oil. J Med Food. 2006, 9:487-490. 10.1089/jmf.2006.9.487

27. Narasimhulu CA, Selvarajan K, Litvinov D, et al.: Anti-atherosclerotic and anti-inflammatory actions of sesame oil. J Med Food. 2015, 18:11-20. 10.1089/jmf.2014.0138

28. Selvarajan K, Narasimhulu CA, Bapputty R, et al.: Anti-inflammatory and antioxidant activities of the nonlipid (aqueous) components of sesame oil: potential use in atherosclerosis. J Med Food. 2015, 18:393-402. 10.1089/jmf.2014.0138

29. Majdalawieh AF, Ro HS: Sesamol and sesame (Sesamum indicum) oil enhance macrophage cholesterol efflux via up-regulation of PPARgamma1 and LXR $\alpha$ transcriptional activity in a MAPK-dependent manner. Eur J Nutr. 2015, 54:691-700. 10.1007/s00394-014-0747-3

30. Karatzi K, Stamatelopoulos K, Lykka M, et al.: Sesame oil consumption exerts a beneficial effect on endothelial function in hypertensive men. Eur J Prev Cardiol. 2013, 20:202-208. 10.1177/2047487312437625

31. Abdel-Daim MM, Taha R, Ghazy EW, et al.: Synergistic ameliorative effects of sesame oil and $\alpha$-lipoic acid against subacute diazinon toxicity in rats: hematological, biochemical, and antioxidant studies. Can J Physiol Pharmacol. 2016, 94:81-88. 10.1139/cjpp-2015-0131

32. Korou LM, Agrogiannis G, Koros C, et al.: Impact of N-acetylcysteine and sesame oil on lipid metabolism and hypothalamic-pituitary-adrenal axis homeostasis in middle-aged hypercholesterolemic mice. Sci Rep. 2014, 4:6806. 10.1038/srep06806

33. Sharma AK, Bharti S, Bhatia J, et al.: Sesamol alleviates diet-induced cardiometabolic syndrome in rats via up-regulating PPARgamma, PPAR $\alpha$ and e-NOS. J Nutr Biochem. 2012, 23:1482-1489. 10.1016/j.jnutbio.2011.09.011

34. Reena MB, Gowda LR, Lokesh BR: Enhanced hypocholesterolemic effects of interesterified oils are mediated by upregulating LDL receptor and cholesterol 7- $\alpha$ - hydroxylase gene expression in rats. J Nutr. 2011, 141:24-30. 10.3945/jn.110.127027

35. Chen WY, Chen FY, Lee AS, et al.: Sesamol reduces the atherogenicity of electronegative L5 LDL in vivo and in vitro. J Nat Prod. 2015, 27:225-233. 10.1021/np500700z

36. Umesha SS, Naidu KA: Vegetable oil blends with $\alpha$-linolenic acid rich Garden cress oil modulate lipid metabolism in experimental rats. Food Chem. 2012, 15:2845-2851. 10.1016/j.foodchem.2012.05.118

37. Vennila L, Pugalendi KV: Efficacy of sesamol on plasma and tissue lipids in isoproterenolinduced cardiotoxicity in Wistar rats. Arch Pharm Res. 2012, 35:1465-1470. 10.1007/s12272- 


\section{2-0817-0}

38. Wu XL, Liou CJ, Li ZY, et al.: Sesamol suppresses the inflammatory response by inhibiting NFkappaB/MAPK activation and upregulating AMP kinase signaling in RAW 264.7 macrophages. Inflamm Res. 2015, 64:577-588. 10.1007/s00011-015-0836-7

39. Sudhakar B, Kalaiarasi P, Al-Numair KS, et al.: Effect of combination of edible oils on blood pressure, lipid profile, lipid peroxidative markers, antioxidant status, and electrolytes in patients with hypertension on nifedipine treatment. Saudi Med J. 2011, 32:379-385.

40. Mensink RP, de Jong A, Lutjohann D, et al.: Plant stanols dose-dependently decrease LDLcholesterol concentrations, but not cholesterol-standardized fat-soluble antioxidant concentrations, at intakes up to $9 \mathrm{~g} / \mathrm{d}$. Am J Clin Nutr. 2010, 92:24-33. 10.3945/ajcn.2009.29143

41. Plat J, van Onselen EN, van Heugten MM, et al.: Effects on serum lipids, lipoproteins and fat soluble antioxidant concentrations of consumption frequency of margarines and shortenings enriched with plant stanol esters. Eur J Clin Nutr. 2000, 54:671-677. 10.1038/sj.ejcn.1601071

42. Faine LA, Diniz YS, Galhardi CM, et al.: Synergistic action of olive oil supplementation and dietary restriction on serum lipids and cardiac antioxidant defences. Can J Physiol Pharmacol. 2004, 82:969-975. 10.1139/y04-092

43. Wilson TA, Ausman LM, Lawton CW, et al.: Comparative cholesterol lowering properties of vegetable oils: beyond fatty acids. J Am Coll Nutr. 2000, 19:601-607. 10.1080/07315724.2000.10718957

44. Namayandeh SM, Kaseb F, Lesan S: Olive and sesame oil effect on lipid profile in hypercholesterolemic patients, which better?. Int J Prev Med. 2013, 4:1059-1062.

45. Ide T, Azechi A, Kitade S, et al.: Comparative effects of sesame seeds differing in lignan contents and composition on fatty acid oxidation in rat liver. J Oleo Sci. 2015, 64:211-222. 10.5650/jos.ess 14182

46. Ahmad S, Yousuf S, Ishrat T, et al.: Effect of dietary sesame oil as antioxidant on brain hippocampus of rat in focal cerebral ischemia. Life Sci. 2006, 12:1921-1928. 10.1016/j.lfs.2006.06.017

47. Abdou HM, Hussien HM, Yousef MI: Deleterious effects of cypermethrin on rat liver and kidney: protective role of sesame oil. J Environ Sci Health. 2012, 47:306-314. 10.1080/03601234.2012.640913

48. Yamashita K, Iizuka Y, Imai T, et al.: Sesame seed and its lignans produce marked enhancement of vitamin E activity in rats fed a low $\alpha$-tocopherol diet. Lipids. 1995, 30:1019_ 1028. 10.1007/BF02536287

49. Sankar D, Rao MR, Sambandam G, et al.: A pilot study of open label sesame oil in hypertensive diabetics. J Med Food. 2006, 9:408-412. 10.1089/jmf.2006.9.408

50. Sankar D, Ali A, Sambandam G, et al.: Sesame oil exhibits synergistic effect with anti-diabetic medication in patients with type 2 diabetes mellitus. Clin Nutr. 2011, 30:351-358.

10.1016/j.clnu.2010.11.005 